Bull. Austral. Math. Soc.

$16 \mathrm{D} 50,16 \mathrm{P} 40$

VOL. $53(1996) \quad[517-525]$

\title{
NOTES ON WEAKLY-SEMISIMPLE RINGS
}

\author{
YIQIANG ZHOU
}

\begin{abstract}
Responding to a question on right weakly semisimple rings due to Jain, LopezPermouth and Singh, we report the existence of a non-right-Noetherian ring $R$ for which every uniform cyclic right $R$-module is weakly-injective and every uniform finitely generated right $R$-module is compressible. We show that a ring $R$ is a right Noetherian ring for which every cyclic right $R$-module is weakly $R$-injective if and only if $R$ is a right Noetherian ring for which every uniform cyclic right $R$-module is compressible if and only if every cyclic right $R$-module is compressible. Finally, we characterise those modules $M$ for which every finitely generated (respectively, cyclic) module in $\sigma[M]$ is compressible.
\end{abstract}

\section{INTRODUCTION AND NOTATION}

All rings $R$ are associative with identity and all modules are unitary right $R$ modules. For a module $M$ and a submodule $N, N \leqslant_{e} M$ denotes that $N$ is essential in $M$. As usual, $E(M)$ and $Z(M)$ indicate the injective hull and the singular submodule of $M$ respectively. The module $M$ is said to be tight (respectively, $R$-tight) if every finitely generated (respectively, cyclic) submodule of $E(M)$ is embeddable in $M$, while $M$ is defined to be weakly-injective (respectively, weakly $R$-injective) if for any finitely generated (respectively, cyclic) submodule $Y$ of $E(M)$ there exists a submodule $X$ of $E(M)$ such that $Y \subseteq X \cong M$ (see [3] and [5]). A module is called a compressible module if it is embeddable in each of its essential submodules [9]. Rings for which all modules are weakly-injective, called right weakly semisimple rings, were introduced by Jain, Lopez-Permouth and Singh [7] and studied in $[1,6,7,11,12]$.

The following characterisations of right weakly-semisimple rings were obtained in $[7]$.

THEOREM 0 . The following are equivalent for a ring $R$ :

(i) $R$ is a right weakly-semisimple ring;

(ii) Every finitely generated $R$-module is weakly-injective and $R$ is right Noetherian;

(iii) Every cyclic $R$-module is weakly-injective and $R$ is right Noetherian;

Received 9th March, 1995

Copyright Clearance Centre, Inc. Serial-fee code: 0004-9729/96 SA2.00+0.00. 
(iv) Every uniform cyclic $R$-module is weakly-injective and $R$ is right Noetherian;

(v) Every uniform finitely generated $R$-module is compressible and $R$ is right Noetherian.

It is open whether or not one may remove the Noetherian condition from any of the equivalent statements (ii) through ( $v$ ) in the above theorem (see [6] or [7]). Here we shall report an example of a ring $R$ which is not a right Noetherian ring but for which every uniform cyclic module is weakly-injective and every uniform finitely generated module is compressible. Motivated by Theorem 0, we characterise the right Noetherian rings $R$ for which every cyclic $R$-module is weakly $R$-injective and the right Noetherian rings $R$ for which every uniform cyclic $R$-module is compressible. These rings are also mentioned as worthy of study in [7]. We shall show that the two classes of rings coincide and each can be characterised as being those rings for which every cyclic module is compressible. We shall call a ring $R$ a right $C C$-ring if $R$ satisfies one of these equivalent conditions. [This terminology has been used differently in [8].] In Section 3, we consider analogues of right weakly semisimple rings and right $C C$-rings to modules. For any module $M$, we denote by $\sigma[M]$ the full subcategory of $\operatorname{Mod}-R$, whose objects are the submodules of $M$-generated modules, and by $E_{M}(N)$ the $M$-injective hull of a module $N$ which is the trace of $M$ in $E(M)$, that is, $E_{M}(N)=\sum\{f(M): f \in \operatorname{Hom}(M, E(N))\}$ (see [14]). We present characterisations for those modules $M$ for which every finitely generated (respectively, cyclic) module in $\sigma[M]$ is compressible. These characterisations extend naturally the corresponding results of right weakly semisimple rings and right $C C$-rings.

\section{The example}

ExAMPLE 1. Let $Q=\prod_{i}^{\infty} F_{i}$, where each $F_{i}=\mathbf{Z}_{2}=\{\overline{0}, \overline{1}\}$, be the full product of rings $\mathbf{Z}_{2}, R$ the subring of $Q$ generated by $\bigoplus_{i=1}^{\infty} F_{i}$ and $1_{Q}$, and $I$ an arbitrary right ideal of $R$. Then $R$ is not a right Noetherian ring and

(a) $\operatorname{Soc}\left(R_{R}\right)=\bigoplus_{i=1}^{\infty} F_{i}$ is the only proper essential right ideal of $R$ and $R / \operatorname{Soc}\left(R_{R}\right)$ is a two-element field;

(b) $R / I$ is $R$-injective if $I \subseteq S o c\left(R_{R}\right)$ and $S o c(R) / I$ is finitely generated;

(c) $R / I$ is $R$-injective if $I \nsubseteq S o c(R)$;

(d) $R / I$ is $R$-injective and simple if $R / I$ is uniform. Therefore every uniform $R$-module is simple and compressible.

ProOF: It is easy to check (a). We let $\pi_{i}(\alpha)$ denote the $i$ th component of the element $\alpha$ in $Q$. Note that, for $\alpha \in Q, \alpha \in R$ if and only if $\pi_{i}(\alpha)=a$ for all but finitely many $i$ 's with $a=\overline{0}$ or $\overline{1}$. 
(b) Suppose that $I \subseteq S o c\left(R_{R}\right)$ and $S o c(R) / I$ is finitely generated. We can write $I=\bigoplus_{i \in V} F_{i}$, where $V$ is a subset of $\mathrm{N}$. Let $f: \operatorname{Soc}\left(R_{R}\right) \longrightarrow R / I$ be an $R$ homomorphism. Clearly $I \subseteq \operatorname{Ker}(f)$, and we may write $\operatorname{Ker}(f)=\bigoplus_{i \in W} F_{i}$, where $V \subseteq W \subseteq \mathbf{N}$. It can be easily checked that the restriction of $f$ on $\bigoplus_{i \in \mathbf{N} \backslash W} F_{i}$ coincides with $\pi \circ \varepsilon$, where $\varepsilon$ is the identity map on $\bigoplus_{i \in \mathbf{N} \backslash W} F_{i}$ and $\pi: R \longrightarrow R / I$ is the canonical homomorphism. Let $\beta$ be in $Q$ satisfying $\pi_{i}(\beta)=\overline{0}$ for precisely $i \in W$, and $=\overline{1}$ if $i \in \mathbf{N} \backslash W$. Since $\operatorname{Soc}(R) / I$ is finitely generated, the set $\mathbf{N} \backslash V$ and hence the set $\mathbf{N} \backslash W$ is finite. Therefore, $\beta$ is in $R$. Define $g: R \longrightarrow R / I$ by $g(1)=\beta+I$. Then $g$ extends $f$.

(c) Suppose that $I \nsubseteq \operatorname{Soc}\left(R_{R}\right)$. Then there exists a finite subset $U$ of $\mathrm{N}$ such that $\alpha \in I$ if and only if $\pi_{i}(\alpha)=0$ for every $i \in U$. So we have $R=I \oplus\left(\bigoplus_{i \in U} F_{i}\right)$ and $R / I \cong \bigoplus_{i \in U} F_{i}$. Therefore, to show $R / I$ is $R$-injective, it suffices to show that each $F_{i}$ is $R$-injective. Let $f: S o c\left(R_{R}\right) \longrightarrow F_{i}$ be a nonzero $R$-homomorphism. Then $\operatorname{Ker}(f)=\bigoplus_{j \neq i} F_{j}$ and $\left.f\right|_{F_{i}}=1$. Define $g: R \longrightarrow F_{i}$ by $\pi_{j}(g(1))=\overline{0}$ if $j \neq i$ and $\pi_{i}(g(1))=\overline{1}$. Then $g$ extends $f$.

(d) Suppose that $R / I$ is uniform. Case 1: $I \nsubseteq \operatorname{Soc}\left(R_{R}\right)$. As above, we have $R=I \oplus\left(\bigoplus_{i \in U} F_{i}\right)$, where $U$ is a finite subset of $\mathbf{N}$. Then $R / I \cong \bigoplus_{i \in U} F_{i}$. Since $R / I$ is uniform, $|U|=1$ and hence $R / I$ is simple. It follows from (c) that $R / I$ is injective. Case 2: $I \subseteq \operatorname{Soc}\left(R_{R}\right)$. Since $R / I$ is uniform, $\operatorname{Soc}\left(R_{R}\right) / I$ must be zero or simple, and hence finitely generated. By $(b), R / I$ is injective. If $I=\operatorname{Soc}(R)$, then $R / I=R / \operatorname{Soc}(R)$ is simple. If $I \subset \operatorname{Soc}(R)$, then $\operatorname{Soc}(R) / I$ is uniform. Thus $I=\bigoplus_{i \neq j} F_{i}$ for some $j$. Then $R / I=[\operatorname{Soc}(R) / I] \oplus(J / I)$, where $J=\left\{\alpha \in R: \pi_{j}(\alpha)=\overline{0}\right\}$. This contradicts the uniformness of $R / I$.

\section{RINGS WHOSE CYCLICS ARE COMPRESSIBLE}

A module $M$ is called a $V$-module if every simple $R$-module is $M$-injective. The following lemma is an easy corollary of Shock [13, Thorem 3.8].

Lemma 2. For a $V$-module $M, M$ is Noetherian if and only if every factor module of $M$ has finitely generated socle.

THEOREM 3. The following are equivalent for a ring $R$ :

(a) Every $R$-module is weakly $R$-injective (or $R$-tight);

(b) $R$ is right Noetherian and every finitely generated $R$-module is weakly $R$-injective (or $R$-tight); 
(c) $R$ is right Noetherian and every cyclic $R$-module is weakly $R$-injective (or $R$-tight);

(d) $R$ is right Noetherian and every uniform cyclic $R$-module is weakly $R$ injective (or $R$-tight);

(e) $R$ is right Noetherian and every uniform cyclic $R$-module is compressible;

(f) Every cyclic $R$-module is compressible.

Proof: $(f) \Rightarrow(e)$. For any simple $R$-module $M$, each $x R \subseteq E(M)$ is embeddable in $M$ since $M \cap x R \leqslant_{e} x R$. This implies that $E(M)$ is simple. Thus $M=E(M)$ is injective. Therefore, $R_{R}$ is a $V$-module. Next we show that every cyclic $R$-module has finitely generated socle. For a cyclic $R$-module $N$, we have a submodule $X$ of $N$ maximal with respect to $\operatorname{Soc}(N) \cap X=0$ and hence $\operatorname{Soc}(N)$ is essentially embeddable in $N / X$. Since $N / X$ is compressible, we have an embedding $N / X \hookrightarrow \operatorname{Soc}(N)$, implying that $N / X$ is a semisimple module. This shows that $\operatorname{Soc}(N) \cong N / X$ is finitely generated. Therefore, by Lemma $2, R$ is a right Noetherian ring.

$(e) \Rightarrow(f)$. Let $M=x R$ be a cyclic module and $N \leqslant_{e} M$. Since $R$ is right Noetherian, $M$ has finite Goldie dimension. Then there exist cyclic uniform submodules $x_{i} R(i=1, \cdots, n)$ of $M$ such that $x_{1} R+\cdots+x_{n} R=x_{1} R \oplus \cdots \oplus x_{n} R \leqslant N \subseteq M$. Therefore, $E(M) \stackrel{f}{\cong} E\left(x_{1} R\right) \oplus \cdots \oplus E\left(x_{n} R\right)$. Write $f(x)=y_{1}+\cdots+y_{n}$ with $y_{i} \in$ $E\left(x_{i} R\right)$. Note that each $y_{i} \neq 0$. Then $M \cong f(M)=\left(y_{1}+\cdots+y_{n}\right) R \subseteq y_{1} R \oplus \cdots \oplus$ $y_{n} R$. Since $x_{i} R \cap y_{i} R \leqslant_{\mathrm{e}} y_{i} R$ and $y_{i} R$ is uniform, we have $y_{i} R \stackrel{g_{i}}{\hookrightarrow} x_{i} R$. Define $g: y_{1} R \oplus \cdots \oplus y_{n} R \longrightarrow x_{1} R \oplus \cdots \oplus x_{n} R$ by $g\left(y_{1} r_{1}+\cdots+y_{n} r_{n}\right)=g_{1}\left(y_{1}\right) r_{1}+\cdots+$ $g_{n}\left(y_{n}\right) r_{n}$ (all $r_{i} \in R$ ). Then $g$ is one-to-one and we have $M \stackrel{\text { gof }}{\rightarrow} N$, showing that $M$ is compressible.

To complete the proof, we note two facts. First, it is straightforward to verify that every cyclic (respectively, uniform cyclic) $R$-module is compressible if and only if every $R$-module (respectively, uniform $R$-module) is $R$-tight. Next, from [6, 2.8], we see that for a ring $R$ for which every cyclic $R$-module has finitely generated socle, an $R$-module $M$ is tight (respectively, $R$-tight) if and only if $M$ is weakly-injective (respectively, weakly $R$-injective). Therefore, the remaining equivalences follow from these facts and the equivalence $(e) \Leftrightarrow(f)$.

From now on, we call a ring $R$ a right $C C$-ring if $R$ satisfies any one of the equivalent conditions in Theorem 3.

It is known that, for a semiprime right Goldie ring $R, R$ is left Goldie if and only if every finitely generated non-singular $R$-module is embeddable in a free module [10] if and only if every finitely generated non-singular $R$-module is compressible $[9,2.2 .15]$. We need the following proposition for the next characterisation of right $C C$-rings.

PROPOSITION 4. The following are equivalent for a semiprime right Goldie ring 
$R$ :

(a) Every cyclic non-singular $R$-module is embeddable in a free module;

(b) Every cyclic non-singular $R$-module is compressible;

(c) $R_{R}$ is $R$-tight.

Proof: The implication $(a) \Rightarrow(b)$ follows from $[15$, Theorem 5], while $(b) \Rightarrow(c)$ is obvious.

$(c) \Rightarrow(a)$. Let $M=x R$ be non-singular. We let $Q$ be the right classical quotient ring of $R$. Then $Q$ is a semisimple ring. Consider the right $Q$-module $M \underset{R}{\bigotimes_{R}} Q$. Clearly $M \bigotimes_{R} Q=(x \otimes 1) Q$ is cyclic. Since $Q$ is a semisimple ring, $M \bigotimes_{R} Q$ is a semisimple $Q$-module, implying that $M \bigotimes_{R} Q$ is of finite length as a $Q$-module. By [4, Exercise 6E, p.104], $M$ has finite Goldie dimension and so there exist uniform cyclic submodules $x_{i} R(i=1, \cdots, n)$ of $M$ such that $x_{1} R+\cdots+x_{n} R=x_{1} R \oplus \cdots \oplus x_{n} R \leqslant_{e} M$. Then $E(M) \stackrel{f}{\cong} E\left(x_{1} R\right) \oplus \cdots \oplus E\left(x_{n} R\right)$. Write $f(x)=y_{1}+\cdots+y_{n}$ with $y_{i} \in E\left(x_{i} R\right)$. We have $M \cong f(x) R=\left(y_{1}+\cdots+y_{n}\right) R \subseteq y_{1} R \oplus \cdots \oplus y_{n} R$. Therefore, to show $(a)$ it suffices to show that every uniform cyclic non-singular right $R$-module is embeddable in a free module. So we may assume that $M=x R$ is uniform. Since $x R$ is non-singular, $x^{\perp}$ is not essential in $R$. Thus, $x^{\perp} \cap I=0$ for some $0 \neq I \subseteq R_{R}$, implying $I \hookrightarrow x R$. Then $E(I) \cong E(x R)$ since $x R$ is uniform. Now it follows from (c) that $x R$ is embeddable in $R$.

Let $R$ be a right Ore-domain but not a left Ore-domain and $T=M_{2}(R)$ be the $2 \times 2$ matrix ring over $R$. Then $T$ is a semiprime right Goldie ring, $T_{T}$ is not $T$-tight [6, Remark 3.7], and $R_{R}$ is $R$-tight.

THEOREM 5. The following are equivalent for a ring $R$ :

(a) $R$ is a right $C C$-ring;

(b) $R$ is a right $Q I$-ring, $R_{R}$ is $R$-tight, and every singular cyclic $R$-module is compressible;

(c) $R$ is semiprime right Goldie, $R_{R}$ is $R$-tight, and every singular cyclic $R$-module is compressible.

Proof: $(a) \Rightarrow(b)$. By Boyle [2], right $Q I$-rings can be characterised as being those right Noetherian rings for which every uniform cyclic module is strongly-prime, where a module $M$ is called strongly-prime if $M$ is contained in every nonzero quasiinjective submodule of $E(M)$. Note that every uniform cyclic module being compressible implies every uniform cyclic module being strongly-prime. Therefore, the implication follows from Theorem 3.

(b) $\Rightarrow(c)$. Obvious. 
(c) $\Rightarrow(a)$. Let $M=x R$ be a cyclic module and $N \leqslant$. There exists a non-singular submodule $K$ of $N$ such that $Z(N) \oplus K \leqslant_{\mathrm{e}} N \subseteq M$. Then $E(M)=$ $E(Z(N)) \oplus E(K)$. Write $x=a+b$, where $a \in E(Z(N))$ and $b \in E(K)$. Since $R$ is right non-singular, $a R$ is singular. Then, by $(c)$, we have $a R \hookrightarrow Z(N)$ since $Z(N) \cap a R \leqslant$ e $a R$. Note that $b R$ is non-singular and hence is compressible by Proposition 4. It follows that $b R \hookrightarrow K$ since $K \cap b R \leqslant_{e} b R$. Therefore, we have $a R \oplus b R \hookrightarrow Z(N) \oplus K \subseteq N$, implying $x R \hookrightarrow N$. Therefore, $M$ is compressible.

\section{MOdUle ANALOgUes OF RIGHT WEAKLY SEMISIMPLE RINGS AND RIGHT $C C$-RINGS}

Definition 6: Let $M$ and $N$ be $R$-modules. $N$ is said to be tight (respectively, $R$-tight) with respect to $M$, if every finitely generated (respectively, cyclic) submodule of $E_{M}(N)$ is embeddable in $N . N$ is said to be weakly-injective (respectively, weakly $R$-injective) with respect to $M$, if for every finitely generated (respectively, cyclic) submodule $Y$ of $E_{M}(N)$ there exists $X \subseteq E_{M}(N)$ such that $Y \subseteq X \cong N$.

Remarks 7. (1) For any generator $M$ in Mod $-R$, a module $N$ is tight (or $R$-tight, or weakly-injective, or weakly $R$-injective, respectively) with respect to $M$ if and only if $N$ is tight (or $R$-tight, or weakly-injective, or weakly $R$-injective, respectively).

(2) A tight (respectively, $R$-tight) module $N$ is tight (respectively, $R$-tight) with respect to $M$ for any $M \in$ Mod-R. But if $R=\mathbf{Z}$ and $M=\mathbf{Z}_{2}$ then $E(M)=\mathbf{Z}_{2}(\infty)$ and $E_{M}(M)=Z_{2}$. Therefore, $M$ is tight with respect to $M$ but not $R$-tight.

(3) A weakly-injective $R$-module may not be weakly $R$-injective with respect to some module $M$. For instance, consider $R=\mathbf{Z}_{4}$ and $M=2 R$. Then $E\left(R_{R}\right)=R$ and $E_{M}(R)=M$. Therefore $R_{R}$ is weakly-injective. Obviously there does not exist $X \subseteq E_{M}(R)$ such that $M \subseteq X \cong R$. Thus, $R_{R}$ is not weakly $R$-injective with respect to $M$.

THEOREM 8. The following are equivalent for a module $M$ :

(a) Every module in $\sigma[M]$ is weakly $R$-injective (or $R$-tight) with respect to $M$;

(b) $M$ is locally Noetherian and every finitely generated module in $\sigma[M]$ is weakly $R$-injective (or $R$-tight) with respect to $M$;

(c) $M$ is locally Noetherian and every cyclic module in $\sigma[M]$ is weakly $R$ injective (or $R$-tight) with respect to $M$;

(d) $M$ is locally Noetherian and every uniform cyclic module in $\sigma[M]$ is weakly $R$-injective (or $R$-tight) with respect to $M$;

(e) $M$ is locally Noetherian and every uniform cyclic module in $\sigma[M]$ is compressible;

(f) Every cyclic module in $\sigma[M]$ is compressible. 
ProOF: $(a) \Rightarrow(b)$. Let $N$ be any cyclic submodule of $M$. For any simple module $X \in \sigma[M]$, we have $X \leqslant_{e} E_{M}(X)$. By $(a)$, every cyclic submodule of $E_{M}(X)$ is embeddable in $X$, and so $X=E_{M}(X)$ is $M$-injective. Therefore, $X$ is $N$-injective. Note that any simple module which is not in $\sigma[M]$ is trivially $N$-injective. This shows that $N$ is a $V$-module. By using the same argument in the proof that $(f) \Rightarrow(e)$ of Theorem 3, we can show that every factor module of $N$ has finitely generated socle. By Lemma 2, $N$ is Noetherian. Therefore, $M$ is locally Noetherian.

$(b) \Rightarrow(c) \Rightarrow(d) \Rightarrow(e)$. Clear.

$(e) \Rightarrow(f)$. Let $N$ be a cyclic module in $\sigma[M]$. Note that $N$ is embeddable in a factor module of a finite direct sum of cyclic submodules of $M$. Since $M$ is locally Noetherian, $N$ is Noetherian and hence has finite Goldie dimension. Now as in the proof that $(e) \Rightarrow(f)$ of Theorem $3, N$ is compressible.

$(f) \Rightarrow(a)$. First we note that every cyclic in $\sigma[M]$ is compressible if and only if every module in $\sigma[M]$ is $R$-tight with respect to $M$. In particular, by the implication $(a) \Rightarrow(b), M$ is locally Noetherian. Let $N$ be a module in $\sigma[M]$ and $Y$ a cyclic submodule of $E_{M}(N)$. Since $N \cap Y \leqslant e Y$, we have an embedding $Y \stackrel{f}{\rightarrow} N \cap Y$ since $Y$ is compressible. Note that $E_{M}(Y)$ is quasi-injective. There exists a homomorphism $g$ : $E_{M}(Y) \longrightarrow E_{M}(Y)$ which extends $f$. Since $M$ is locally Noetherian, $Y$ is Noetherian and hence has finite Goldie dimension. It follows that $g$ is an isomorphism. Note that $E_{M}(N)=E_{M}(Y) \oplus Z$ for some $Z \subseteq E_{M}(N)$. If we define $h: E_{M}(N) \longrightarrow E_{M}(N)$ by $h(a+b)=g(a)+b$ for all $a \in E_{M}(Y)$ and $b \in Z$, then $h$ is an isomorphism which extends $g$. Let $X=h^{-1}(N)$. Then $Y \subseteq X \cong N$. Therefore, $N$ is weakly $R$-injective.

THEOREM 9. The following are equivalent for a module $M$ :

(a) Every module in $\sigma[M]$ is weakly-injective (or tight) with respect to $M$;

(b) $M$ is locally Noetherian and every finitely generated module in $\sigma[M]$ is weakly-injective (or tight) with respect to $M$;

(c) $M$ is locally Noetherian and every cyclic module in $\sigma[M]$ is weakly injective (or tight) with respect to $M$;

(d) $M$ is locally Noetherian and every uniform cyclic module in $\sigma[M]$ is weakly injective (or tight) with respect to $M$;

(e) $M$ is locally Noetherian and every uniform finitely generated module in $\sigma[M]$ is compressible;

(f) Every finitely generated module in $\sigma[M]$ is compressible.

Proof: $(a) \Rightarrow(b)$. By Theorem 8 .

$(b) \Rightarrow(c) \Rightarrow(d) \Rightarrow(e)$. Clear.

$(e) \Rightarrow(f)$. Let $N$ be a finitely generated module in $\sigma[M]$ and $P \leqslant_{e} N$. Since $M$ is 
locally Noetherian, $N$ is Noetherian and hence has finite Goldie dimension. Then there exist cyclic uniform submodules $x_{i} R$ of $N(i=1, \cdots, n)$ such that $x_{1} R+\cdots+x_{n} R=$ $x_{1} R \oplus \cdots \oplus x_{n} R \leqslant \mathrm{e} P$. Then $E_{M}(N) \stackrel{f}{\cong} E_{M}\left(x_{1} R\right) \oplus \cdots \oplus E_{M}\left(x_{n} R\right)$. Since $N$ is finitely generated, there exist finitely generated submodules $Y_{i}$ of $E_{M}\left(x_{i} R\right)$ such that $f(N) \subseteq Y_{1} \oplus \cdots \oplus Y_{n}$. Each $Y_{i}$ is a finitely generated uniform module and hence compressible by $(e)$. Therefore, we have an embedding $Y_{i} \stackrel{g_{i}}{\rightarrow} Y_{i} \cap x_{i} R$. Define $g: Y_{1} \oplus \cdots \oplus Y_{n} \longrightarrow x_{1} R \oplus \cdots \oplus x_{n} R$ by $g\left(y_{1}+\cdots+y_{n}\right)=g_{1}\left(y_{1}\right)+\cdots+g_{n}\left(y_{n}\right)$ for all $y_{i} \in Y_{i}$. Then $g$ is one-to-one and we have $N \stackrel{g \circ f}{\rightarrow} P$, showing that $N$ is compressible. $(f) \Rightarrow(a)$. Similar to the proof that $(f) \Rightarrow(a)$ in Theorem 8 .

\section{REFERENCES}

[1] A. Al-Huzali, S.K. Jain and S.R. Lopez-Permouth, 'On the weak relative-injectivity of rings and modules', in Noncommutative ring theory, Lecture Notes in Math. 1448 (Springer-Verlag, Berlin, Heidelberg, New York, 1990), pp. 93-98.

[2] A.K. Boyle, 'Injectives containing no proper quasi-injective submodules', Comm. Algebra 4 (1976), 775-785.

[3] J.S. Golan and S.R. Lopez-Permouth, 'QI-filters and tight modules', Comm. Algebra 19 (1991), 2217-2229.

[4] K.R. Goodearl and R.B. Warfield Jr., An introduction to noncommutative Noetherian rings (London Math. Soc., Cambridge Univ. Press, Cambridge, 1989).

[5] S.K. Jain and S.R. Lopez-Permouth, 'Rings whose cyclics are essentially embeddable in projective modules', J. Algebra 128 (1990), 257-269.

[6] S.K. Jain and S.R. Lopez-Permouth, 'A survey on the theory of weakly-injective modules', in Computational Algebra, Lecture Notes in Pure and Appl. Math. 151 (Marcel Dekker, New York, 1994), pp. 205-232.

[7] S.K. Jain, S.R. Lopez-Permouth and S.Singh, 'On a class of QI-rings', Glasgow Math. J. 34 (1992), 75-81.

[8] S.K. Jain and S. Mohamed, 'Rings whose cyclic modules are continuous', J. Indian Math. Soc. 42 (1978), 197-202.

[9] A.V. Jategaonkar, Localization in Noetherian rings (Cambridge University Press, Cambridge, London, New York, 1986).

[10] L. Levy, 'Torsion-free and divisible modules over non-integral-domains', Canad. J. Math. 15 (1963), 132-151.

[11] S.R. Lopez-Permouth, 'Rings characterized by their weakly-injective modules', Glasgow Math. J. 34 (1992), 349-353.

[12] S.R. Lopez-Permouth, S.T. Rizvi and M.F. Yousif, 'Some characterizations of semiprime Goldie rings', Glasgow Math. J. 35 (1993), 357-365.

[13] R.C. Shock, 'Dual generalizations of the Artinian and Noetherian conditions', Pacific J. Math. 54 (1974), 227-235.

[14] R. Wisbauer, 'Generalized co-semisimple modules', Comm. Algebra 8 (1990), 4235-4253. 
[15] Y. Zhou, 'Strongly compressible modules and semiprime right Goldie rings', Comm. Algebra 21 (1993), 687-698.

Department of Mathematics University of British Columbia Vancouver BC V6T 122 Canada
Current address:

Department of Mathematics and Statistics Memorial University of Newfoundland

St. John's, NF

A1C 5S7 Canada 